\title{
WikiProject Medicine making progress
}

I $\mathrm{n}$ the 14 years since Wikipedia launched, it has become one of the most popular sources of health information online. Although some doctors decry its crowd-sourced content as unreliable, studies indicate that many use it. Some even cite Wikipedia in their peer-reviewed journal articles, despite the availability of evidence-based sources, according to a $2014 B M J$ study.

In 2004, an international group of physicians and other medical professionals conceded the popularity of the Wikipedia juggernaut and formed the voluntary WikiProject Medicine to improve its medical content.

"It's easier to fix Wikipedia than it is to convince the half a billion people each month who use it, not to," says Dr. James Heilman, president of the nonprofit Wiki Project Med Foundation and a clinical assistant professor at the University of British Columbia, Vancouver.

Research that Heilman recently did with Microsoft (not yet published) showed that in the three countries most affected by the Ebola outbreak, the single most-used online source of information about the disease was Wikipedia.

Not only is Wikipedia consulted, but more than 2000 websites mirror its content, including Facebook.

The hazards of using Wikipedia for medical information are well known and were recounted in a $2014 B M J$ study, headed by Dr. M. Dylan Bould, an assistant professor at the University of Ottawa. Chief among them is that anyone with access to the Internet can alter Wikipedia articles and insert misinformation, intentionally or not, Bould's group said.

Working to reduce these hazards is WikiProject Medicine's current group of about 270 core editors (defined as those who have edited at least 250 articles in a year). They work with translation groups to cover all 287 languages in which Wikipedia appears. Heilman began volunteering with the project in 2007 and is now the most prolific of its medical editors. In fact, he cut back on clinical work to devote more time to editing.

"Wikipedia isn't nearly as good as it could be yet, but the efforts myself and my fellow Wikipedians have put in

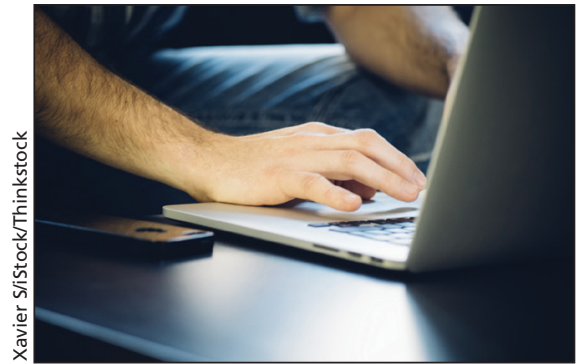

The public and physicians often rely on Wikipedia for health information. WikiProject Medicine aims to improve the content.

over the years have definitely improved the quality of its content," says Heilman, who is also the head of the emergency department at East Kootenay Regional Hospital in Cranbrook, BC.

In one case, the editors successfully published a Wikipedia clinical review on dengue fever in the peer-reviewed journal Open Medicine (which has since ceased operation).

The group is also establishing collaborations with universities, the Cochrane Collaboration, the United States Agency for Healthcare Research and Quality, and the World Health Organization, he says.

Heilman is also conducting a study with Samir Grover, an assistant professor of medicine in the Division of Gastroenterology at the University of Toronto, to explore whether Wikipedia has a place as a medical text. They are enrolling 50 to 100 first- and second-year medical students in a study to see how they fare on an open-book standardized test using Wikipedia, UpToDate (evidence-based point-of-care clinical decision software) or electronic medical textbooks.

The researchers will compare each group's test scores, as well as the content areas where they make mistakes. For example, Wikipedia is thought to be weak in treatment, so they'll want to see whether the students make mistakes in areas where each resource is known to be weak.

Grover says Wikipedia may also be better suited to the way adults prefer to learn, that is, going to a resource where they can rapidly find an answer to a problem. - Terry Murray, Toronto, Ont.

CMAJ 2015. DOI:10.1503/cmaj.109-4982 